Check for updates

Cite this: Phys. Chem. Chem. Phys., $2018,20,27528$

Received 25th July 2018, Accepted 18th September 2018

DOI: $10.1039 / c 8 c p 04741 c$

rsc.li/pccp

\section{The excess electron in polymer nanocomposites $\dagger$}

\begin{abstract}
Fernan Saiz (D) and Nick Quirke (iD *
We have used ab initio molecular dynamics and density-functional theory (DFT) calculations at the B3LYP/6-31G** level of theory to evaluate the energy and localisation of excess electrons at a number of representative interfaces of polymer nanocomposites. These modelled interfaces are made by combining liquid water and amorphous slabs of polyethylene and silica. The walls of the amorphous silica slabs are built with two surface chemistries: $Q^{4}$ or fully-dehydroxylated and $Q^{3} / Q^{4}$ or partiallyhydroxylated with a silanol content between 1.62 and 6.86 groups per $\mathrm{nm}^{2}$. Our results indicate that in silica/polyethylene systems an excess electron would sit at the interface with energies between $-1.75 \mathrm{eV}$ with no hydroxylation and $-0.99 \mathrm{eV}$ with the highest silanol content. However, in the presence of a free water film, the chemistry of the silica surface has a negligible influence on the behaviour of the excess electron. The electron sits preferentially at the water/vapour interface with an energy of minus a few tenths of an $\mathrm{eV}$. We conclude that the moisture content in a wet polymer nanocomposite has a profound influence on the electron trapping behaviour as it produces much lower trapping energies and a higher excess-electron mobility compared to the dry material.
\end{abstract}

\section{Introduction}

Electron transfer underpins technologies such as photovoltaics, ${ }^{1,2}$ organic thin film transistors, light-emitting diodes, ${ }^{3}$ photocatalysis, ${ }^{4}$ DNA based molecular electronics, ${ }^{5,6}$ as well as energy transfer in nature ${ }^{7}$ including radiation damage and repair. ${ }^{8,9}$ The injection of excess electrons plays a major role in the performance of electrical insulation with significant economic consequences..$^{10}$ Polymers are commonly employed as insulators in electric and electronic devices such as capacitors, ${ }^{11-13}$ transistors, ${ }^{14,15}$ fuel cell membranes, ${ }^{16,17}$ and in high-voltage cables. ${ }^{18}$ When polymers are used for highvoltage cables their insulating properties such as the electrical conductivity or breakdown strength degrade over time due to exposure to heat, light, moisture, surfactants, mechanical stress, and high electric fields. ${ }^{19,20}$ This degradation process is thought to be related to the trapping processes of charge carriers, the so-called space charge. ${ }^{19}$

It has been suggested that the insulating properties of polymers can be improved by the addition of nanoparticles to form nanocomposites: a homogeneously-dispersed blend of a dielectric material with a filler whose particles have radii of up to a few tens of a nanometre. ${ }^{21}$ Polymer nanocomposites made by blending a polymer with oxide nanoparticles have been reported to have higher effective permittivities, ${ }^{22,23}$ and enhanced electrical breakdown strength ${ }^{24}$ than those of the base polymer.

Department of Chemistry, Imperial College, London, UK. E-mail: n.quirke@ic.ac.uk

$\dagger$ Electronic supplementary information (ESI) available. See DOI: $10.1039 / \mathrm{c} 8 \mathrm{cp} 04741 \mathrm{c}$
It is thought this enhancement is a consequence of a reduction of injected (excess) electron mobility caused by trapping at the new interfaces created by the presence of nanoparticle surfaces, in addition to those already present in the base polymer (through nanovoids ${ }^{25}$ and chemical defects and impurities ${ }^{26}$ ). In polyethylene, the addition of nanoparticles has been shown to suppress space charge. ${ }^{27}$

A common choice of nanoparticle additive is silicon oxide $\left(\mathrm{SiO}_{2}\right)$. Thermally grown oxides on silicon and silicon carbide supports exhibit high electric breakdown field strengths of up to $9.2 \mathrm{MV} \mathrm{cm}{ }^{-1} \cdot{ }^{28}$ Photon stimulated tunnelling (PST) of electrons at the $\mathrm{Si} / \mathrm{SiO}_{2}$ interface show the presence of very deep electron traps of $2.77 \pm 0.05 \mathrm{eV}$ (below the conduction band). ${ }^{29}$ Electron trapping is known to have a dramatic effect on the performance and reliability of electronic devices employing silicon dioxide as gate insulators and in charge trap flash memory devices. ${ }^{30,31}$ While it is clear that addition of silica nanoparticles changes the electrical characteristics of polymer nanocomposites, there is some doubt as to whether these effects are due to the presence of nanoparticles (with or without a surface coating) or due to water adsorption or entrained solvent associated with the creation of the nanocomposite. ${ }^{32}$

Therefore, the goal of this work is to study the properties of excess electrons at a number of interfaces relevant to nanocomposites by combining amorphous polyethylene, water, and amorphous silica phases using density functional theory (DFT). Our aim is to understand at a fundamental level the nanoscopic processes underlying the experimental data discussed above, in particular the possible role of water. This DFT 
work significantly extends our previous studies of pure polyethylene $e^{25,26,33-35,41}$ to encompass mixtures.

In the past DFT has been used to study the degree of localisation of excess electrons at polyethylene interfaces ${ }^{36,37}$ and in bulk ${ }^{38,39}$ by assuming that the excess electron can be described by Kohn-Sham orbitals. However, it has been unclear to what extent the use of these orbitals could be justified ${ }^{40}$ to model excess electrons, especially when employing hybrid functionals as the Koopmans' theorem is only valid for closedshell Hartree-Fock theory. Nevertheless, we have recently used the all-electron CRYSTAL $17^{59}$ code at the B3LYP level of theory to compare the representation of an excess electron in polyethylene in bulk and its vacuum interfaces by the lowestunoccupied molecular orbital (LUMO) of a $N$ electron system and the highest-occupied molecular orbital (HOMO) of an $N+1$ system (the +1 electron balanced by a uniform background charge). ${ }^{41}$ These two representations have also been compared with the single-electron Lanczos behaviour ${ }^{34}$ employing an excess electron-polyethylene pseudopotential fitted ${ }^{34}$ to experimental data for the bottom of the conduction band of alkanes measured with respect to vacuum. Although both orbitals localise excess electrons at similar positions in the interface and have similar localisation lengths, the excess electron energy predicted by the $\operatorname{LUMO}(N)$ corrected to a zero at the vacuum level, agrees best with the Lanczos' for the larger vacuum gaps ${ }^{41}$ and hence will be used in this work.

The surface chemistry of nanoparticles used to create nanocomposites affects the dispersion of nanoparticles in a polymer matrix as well as the measured electrical properties. Thus, we vary the silica surface chemistry in this study to make it more or less hydrophilic. A silica surface can be characterised by the coordination number $\mathrm{Q}^{n}$ of the surface silicon atoms. We create both $\mathrm{Q}^{3}$ and $\mathrm{Q}^{4}$ amorphous surfaces. On a $\mathrm{Q}^{4}$ surface all dangling silicon atoms are bridged with four oxygen neighbours, similarly, on a $\mathrm{Q}^{3}$ surface silicon atoms are connected with three oxygen neighbours and a hydroxyl group -OH (silanol). The $\mathrm{Q}^{4}$ surface is obtained experimentally by calcination of $\mathrm{Q}^{3}$ and $\mathrm{Q}^{2}$ surfaces at $900 \mathrm{~K}^{42}$ and is hydrophobic with a heat of immersion of $22 \mathrm{mN} \mathrm{m}^{-1}$ whereas the silanated surfaces are hydrophilic. ${ }^{43}$ We also consider the effect of adding a free water film to the silica surface and the role of silanols formed spontaneously in the $a b$ initio simulations.

The manuscript is organised as follows. In Section II, we briefly describe the methods employed to build the bulk and interfacial systems and the parameterisation of the DFT calculations. Given the large amount of detail, we present the full methodology in the ESI. $\dagger$ In Section III, we present the degrees of localisation and energies of excess electrons in bulk systems and for the interfacial systems of amorphous polyethylene and water, amorphous polyethylene and silica, and water and amorphous silica. In Section IV, we compare the excess electron properties in bulk and at interfaces, we also predict the valence and conduction band offsets at the interfaces. Finally, Section V concludes our main findings and looks at the implications for future work.

\section{Simulation methods}

In this section we describe the main procedures used to prepare the systems of amorphous polyethylene, amorphous silica, and liquid water, and their interfaces as well as the $a b$ initio calculations to obtain their excess-electron properties. Full details are given in Section SI of the ESI. $\dagger$

Slabs of amorphous silica are prepared in a two-stage process. In the first stage, we use the classical molecular package LAMMPS ${ }^{44}$ to generate a bulk system made with $3 \times 3 \times 2$ unit cells in the beta-cristobalite lattice. We then follow a melting-quench process to yield an amorphous cube with a density of $2.18 \mathrm{~g} \mathrm{~cm}^{-3}$, close to the experimental value of $2.20 \mathrm{~g} \mathrm{~cm}^{-3}$. ${ }^{45}$ The resulting configuration is then imported to the Materials Studio package, ${ }^{46}$ where a vacuum gap of $2 \mathrm{~nm}$ is imposed in the $z$-direction to create two surfaces. We then bridge a small number of $\mathrm{Si}$ atoms to four $\mathrm{O}$ atoms on the surface and in the inner regions of the slab. We next optimise the geometry with the COMPASS $2^{47}$ force field available in the Forcite Module and then with the DFTB+ Module and finally with $a b$ initio package $\mathrm{CP}_{2} \mathrm{k}^{48}$ in its QUICKSTEP ${ }^{49}$ implementation. CP2k uses GTH pseudopotentials ${ }^{50,51}$ and double- $\zeta$ basis sets with polarization functions (DZVP) as well as Grimme scheme ${ }^{52}$ to include long-range forces. This bridging and optimisation is repeated until the surface is stable at which point we run an $a b$ initio molecular dynamics simulation with CP2k with a timestep of $0.25 \times 10^{-3} \mathrm{ps}$. The final $\mathrm{Q}^{4}$ (no dangling silanol Si-OH groups) surface has a cross section of $1.578 \mathrm{~nm} \times 1.572 \mathrm{~nm}$ in the $y$ and $z$ directions and a length of $2.475 \mathrm{~nm}$ in the $x$-direction. We believe that this length is sufficiently long to reproduce bulk-like conditions in the inner layers of this silica slab as Goumans et al. ${ }^{53}$ showed that a slab of quartz-silica with a thickness of $\mathbf{1 . 1 2 5} \mathrm{nm}$ is sufficiently large to represent bulk-like behaviour in the innermost layers. We use this $\mathrm{Q}^{4}$ surface to create others with $\mathrm{Q}^{3} / \mathrm{Q}^{4}$ chemistries, in which a few $\mathrm{Si}-\mathrm{O}$ are broken in the $\mathrm{Q}^{4}$ surface and replaced with silanol Si-OH groups with concentrations of up to 6.86 groups per $\mathrm{nm}^{2} \cdot{ }^{54}$ Once the silanol groups are built, the slabs are optimised with $\mathrm{CP} 2 \mathrm{k}$ and run with ab initio molecular dynamics again.

The amorphous polyethylene systems are also prepared using the Materials Studio software using four chains of 40 carbons with the COMPASS2 force field. The amorphous solid has the same cross section as the amorphous silica slab and a depth of $1.76 \mathrm{~nm}$. Chains are not allowed to cross the boundaries in the $x$ direction to avoid splitting the chains when a vacuum gap of $2.0 \mathrm{~nm}$ is imposed (see later) though periodic boundary conditions are still applied in all three directions. These configurations are then relaxed with $\mathrm{CP} 2 \mathrm{k}$ with the local-density approximation (LDA) and the long-range forces are described by the Dion-RydbergSchroeder-Langreth-Lundqvist (DRSLL) nonlocal van der Waals density functional. ${ }^{55}$ After the structure is optimised, we run a short $a b$ initio molecular dynamics simulation of 1 ps to further relax the structure.

Finally, ensembles of liquid water at $300 \mathrm{~K}$ are first prepared using the TIP04/2005 model, ${ }^{56}$ which gives excellent density 
predictions at $278 \mathrm{~K}$. The system is composed of 150 molecules, occupying a parallelepiped region with the same cross section as the silica and polyethylene samples. We next run an ab initio molecular dynamics simulation with CP2k for 25 ps with a timestep of $0.25 \times 10^{-3} \mathrm{ps}$ to relax the bond lengths with the $\mathrm{PBE}^{57}$ functional revised for small molecules (revPBE ${ }^{58}$ ) in the bulk and, with a vacuum gap of $2.0 \mathrm{~nm}$, to create a water/vapour interface. We have used the revPBE functional because from simulations with a cubic system of 343 molecules at constant temperature $300 \mathrm{~K}$ and pressure of $1 \mathrm{bar}$, we have found that this revised form improves significantly the agreement with the experimental density with respect to the original parameterisation PBE: the disagreement is only $3.9 \%$ using revPBE, whereas it deviates up to $13.4 \%$ with PBE. Note though that the high computational cost of these ab initio molecular dynamics calculations restricts the length of time for which we can simulate these water systems. One ps of simulated time with CP2k needs to be run on 64 processors for nearly 10 hours for the bulk phase and 13 hours with the $2.0 \mathrm{~nm}$ vacuum gap.

Once the pure systems have been prepared, we build interfaces of water/polyethylene, silica/polyethylene, and silica/ water/silica. The first is prepared with no vacuum using the revPBE functional and Grimme scheme. The second is prepared with and without a $2.0 \mathrm{~nm}$ vacuum gap, which requires the LDA/DRSLL setup for the former and the $\mathrm{PBEsol}^{59} /$ Grimme for the latter. The use of LDA is justified because the polymer chains evaporate after a few ps of simulation. The third type of interface is prepared with the same vacuum gap and employs the revPBE functional. Once the computational setup is ready, the geometry of each two-component system is optimised with CP2k to avoid undesirable orbital overlap between the atoms at each surface. Thanks to this optimisation, the simulations require a shorter time to equilibrate. The systems are run in C2PK for 7.5 ps for polyethylene/silica and $15 \mathrm{ps}$ for polyethylene/water and $15 \mathrm{ps}$ for water/silica/water to obtain a sufficient number of configurations with a timestep of $0.25 \times 10^{-3} \mathrm{ps}$.

The resulting configurations from the $a b$ initio molecular dynamics run are then used by the all-electron CRYSTAL17 DFT code ${ }^{60}$ to obtain their excess-electron properties. The DFT calculations are carried out using the hybrid B3LYP exchangecorrection functional, which as shown in our previous work ${ }^{41}$ is able to reproduce the experimental band gap of crystalline polyethylene and the energy and degree of spatial localisation of excess electrons in amorphous polyethylene. A standard all-electron $6-31 \mathrm{G}^{* * 61,62}$ basis set is used to represent the local atomic orbitals in terms of primitive Cartesian Gaussian functions. Polarization functions (p-functions for hydrogens and d-functions for carbons, oxygens, and silicons) are used to ensure that the orbitals can distort from their original atomic symmetry, and to adapt to the molecular surroundings leading to a better prediction of the total energy of the system with high hydrogen content. ${ }^{63}$ The size of the systems allows us to restrict the reciprocal space integrations to the $\Gamma$-point of the Brillouin zone and ground-state energy convergence is enforced at $1 \times 10^{-6} \mathrm{Ha}$. We have used the default parameters in CRYSTAL17 to calculate the two-electron coulombic and exchange integrals.
Periodic boundary conditions are imposed on the $x, y, z$ directions in systems with no vacuum using the keyword CRYSTAL and on the $x$ and $y$ directions in systems with a $2.0 \mathrm{~nm}$ vacuum gap using the keyword SLAB. We refer hereinafter as '3D-periodic' to the first type of simulations and '2D-slab' to the second. We use this second type of calculations in systems where surface dipoles are present, such as liquid water or silica slabs with hydroxylated walls, to obtain a well-defined vacuum level, where the zero of the electrostatic potential $V_{\mathrm{z}}$ is defined by the CRYSTAL code in such a way that $V_{\mathrm{z}}(+\infty)=-V_{\mathrm{z}}(-\infty)$. See, for example, the case with a slab of amorphous silica with a silanol surface concentration of $1.61 \mathrm{~nm}^{-2}$ in Fig. S1 in the ESI. $\dagger$ In this case, the permanent dipole in the silica slab divides the space in two parts, with higher and lower potential, having two equally valid vacuum states. An electron extracted from the material will chose the side with a positive potential to have a negative (minimum) potential energy in the vacuum. Hence, we correct the energies of the LUMO using

$$
\mathrm{LUMO}_{\text {corrected }}=\mathrm{LUMO}_{\text {uncorrected }}-e\left\langle V_{z}\right\rangle_{\text {vacuum }},
$$

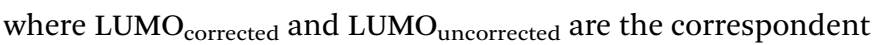
corrected and uncorrected energies of this orbital, $e$ the electron's charge, and $\left\langle V_{z}\right\rangle_{\text {vacuum }}$ is the positive average of the electrostatic potential. For systems with no dipoles, such as slabs of amorphous polyethylene or silica with no silanols, the LUMO energies are corrected with the following expression:

$$
\begin{aligned}
\mathrm{LUMO}_{\text {corrected }}= & \mathrm{LUMO}_{\text {uncorrected }}-\left(E_{\mathrm{band} 1, \text { bulk-like }}-E_{\mathrm{band} 1, \mathrm{bulk}}\right) \\
& -e\left\langle V_{z}\right\rangle_{\text {vacuum }},
\end{aligned}
$$

where the difference between $E_{\text {band1,bulk-like }}$ and $E_{\text {band1,bulk }}$ corresponds the core-level shift of the energies of the band 1 in the bulk case and the bulk-like region from a second simulation with a $2.0 \mathrm{~nm}$ vacuum gap with a $\mathrm{Q}^{4}$ surface. Eqn (2) is also applied to obtain excess electron energies for amorphous polyethylene slabs.

\section{Results and discussion}

\section{1. Pure systems of silica and water}

Before studying the interfacial systems, we first analyse the behaviour of excess electrons in bulk water and silica, as represented by the LUMO of our $N$-electron system. This analysis complements our recent work on bulk amorphous polyethylene and its vacuum interfaces. ${ }^{41}$ In bulk polyethylene an excess electron localises in naturally occurring nanovoids ${ }^{25}$ with radii less than $0.4 \mathrm{~nm}$. In the presence of large empty gaps between planar amorphous slabs, the excess electron localises on the unfilled side of the polyethylene surface, its density peaking at $0.2 \mathrm{~nm}$ into the vacuum with a localisation length of $0.34 \mathrm{~nm}$ and an energy of around $-0.2 \mathrm{eV}$ in good agreement with single-electron methods.

For pure silica, a 3D-periodic calculation with CRYSTAL17 predicts that $80 \%$ of the LUMO's charge sits in a small cavity between atomic rings in the bulk phase as shown in Fig. 1. 

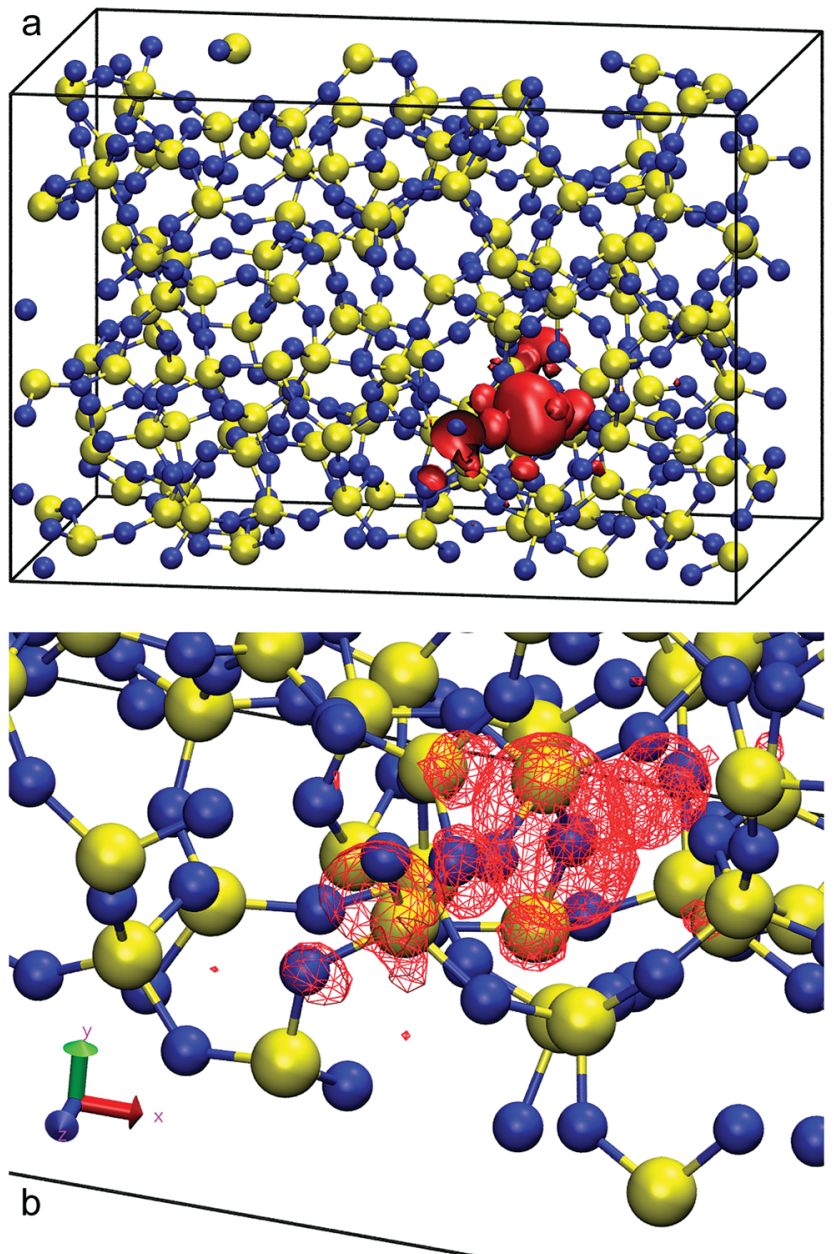

Fig. 1 Red isosurfaces show electron charge densities of $14.05 \times$ $10^{-4}$ electron per Bohr ${ }^{3}$ of the LUMO's $80 \%$ charge of amorphous silica, occupying a cavity between rings (a). Panel (b) shows a zoomed view of the cavity occupied by the LUMO. Silicon atoms are painted in yellow and oxygen in blue.

We obtain a corrected DFT excess electron energy of $-1.54 \mathrm{eV}$ for the $3 \mathrm{D}$-periodic and $-1.43 \mathrm{eV}$ for the $2 \mathrm{D}$-slab calculations (see Table 1). This strong localisation in the bulk of amorphous silica has been also observed in other DFT studies using plane waves ${ }^{64}$ which predict a comparable excess electron localisation energy of $-1.25 \mathrm{eV}$. Our 2D-slab calculations allow us to investigate the effect of adding silanol groups on the silica walls. The results in Table 1 indicate the LUMO energy increases with increasing surface hydroxylation, which suggests that the electron prefers to move away from the hydroxyl groups. This preference has been also observed in polycrystalline $\mathrm{MgO}$, where hydroxyl groups form shallower and more diffuse electron traps than those created on the dehydroxylated surface. ${ }^{65}$

Turning now to liquid water, Fig. 2 illustrates the distribution of $80 \%$ of the LUMO charge in the bulk phase with a 3D-periodic calculation and with vacuum in a $2 \mathrm{D}$-slab simulation. In both cases, the electron with $80 \%$ of its charge is found between 0 and 25 ps in a region that encloses 46 molecules for the bulk and 42 molecules for the ensemble with a vapour (vacuum) interface. These two specific volumes are similar to those reported using a Lanczos algorithm, where the wavefunction overlapped around 37 water molecules within two 3D-periodic cubic simulation cells of $1.817 \mathrm{~nm}$ (200 molecules) and $2.464 \mathrm{~nm}$ (499 molecules). ${ }^{66}$ In the presence of a water interface, the excess electron sits at the interface as shown by the averaged profiles along the $z$-direction plotted in Fig. 3. Our simulations predict a diffusion coefficient for the excess electron of $1.16 \times$ $10^{-9} \mathrm{~m}^{2} \mathrm{~s}^{-1}$ for the 3D-periodic system and an order of magnitude higher at $10.68 \times 10^{-9} \mathrm{~m}^{2} \mathrm{~s}^{-1}$ for the $2 \mathrm{D}$-slab with vacuum (see Fig. S2 and S3 in the ESI $\dagger$ ), which compare reasonably well with the experimental measurement of $4.90 \times 10^{-9} \mathrm{~m}^{2} \mathrm{~s}^{-1}$ at $298 \mathrm{~K}$ for bulk water. ${ }^{67}$

As previously discussed, the ground-state energy of the excess electron simulations is approximated as that of the $\operatorname{LUMO}(N)$. By averaging the energies of this orbital every 0.25 ps between 10.0 and $25.0 \mathrm{ps}$, we find an excess-electron energy of -0.12 $( \pm 0.30) \mathrm{eV}$ in a $2 \mathrm{D}$-slab simulation with its centre of charge located at $z=-4.09 \mathrm{~nm}$ from the centre of mass of the 150molecule system. With 200 molecules, this energy increases slightly to $-0.03( \pm 0.37) \mathrm{eV}$ but note the large standard deviation. Our predictions are in good agreement with those obtained with single-electron pseudopotential methods and experiments. For instance, Turi et al. ${ }^{68}$ found that the excess electron localises in cavities of bulk water with an average energy of $-0.23 \mathrm{eV}$ averaging over 500 configurations and 500 molecules. His predictions and ours compare well with experimental measurement of $-0.12 \mathrm{eV}$ (see Table 1). Note that, one advantage of using CRYSTAL17 with respect to pseudopotential methods, is that we can extract the energy of all electronic bands, including the HOMO. Doing so, we obtain an electronic band gap of $6.04( \pm 0.39) \mathrm{eV}$ with 150 molecules and $5.53( \pm 0.59) \mathrm{eV}$ with 200 molecules using 2D-slab water simulations, which underestimate the experiments by around $1.2 \mathrm{eV}$. This difference may arise from the presence of the interface or be reduced using more accurate techniques such as GW. Note relying strictly on a pure generalised gradient approximation (GGA) produces worse predictions. ${ }^{69}$ Of course, we can always tune the contribution of the Hartree-Fock exchange in B3LYP or other hybrid DFT functionals to obtain the experimental result.

\section{2. Interfaces of amorphous polyethylene and water}

Taking as a reference the behaviour of excess electrons in pure water, we now analyse the interfacial system made by combining water with a slab of amorphous polyethylene. Unfortunately, in this case we cannot calculate the energy of the LUMO from a 2D-slab calculation with a system made of water, polyethylene, and vacuum as it requires running ab initio molecular dynamics with CP2k. This CP2k calculation requires in turn choosing an appropriate exchange-correlation functional and here we find that any PBE functional will ultimately cause the polymer chains to evaporate in the presence of vacuum. The alternative is to use LDA, which we find severely overestimates the density of water at $300 \mathrm{~K}$. We therefore study interfacial systems of amorphous polyethylene and water with revPBE, but without a vacuum phase, thus preventing the disintegration of the polyethylene 
Table 1 Energies of the lowest unoccupied molecular orbital $\left(E_{\mathrm{LUMO}}\right)$, and the band gap energy $\left(E_{\text {gap }}\right)$ of one-compound systems. DFT values are made with CRYSTAL17 at the B3LYP level of theory. Experimental measurements of the energies of excess electrons $\left(E_{\exp }\right)$ and band gaps are also shown for comparison

\begin{tabular}{|c|c|c|c|}
\hline System & Calculation details & $E_{\mathrm{LUMO}} E_{\exp }(\mathrm{eV})$ & $E_{\text {gap }}(\mathrm{eV})$ \\
\hline $\mathrm{Q}^{4}$ surface $\mathrm{SiO}_{2}+2.0 \mathrm{~nm}$ vacuum & 3D-periodic & -1.43 & 5.84 \\
\hline $\mathrm{Q}^{4}$ surface $\mathrm{SiO}_{2}+2.0 \mathrm{~nm}$ vacuum & 2D-slab & -2.06 & 5.75 \\
\hline $\mathrm{Q}^{3} \mathrm{Q}^{4}$ surface $\mathrm{SiO}_{2}$ with $[\mathrm{SiOH}]=3.22 \mathrm{~nm}^{-2}+2.0 \mathrm{~nm}$ vacuum & 2D-slab & -1.33 & 6.20 \\
\hline $\mathrm{Q}^{3} \mathrm{Q}^{4}$ surface $\mathrm{SiO}_{2}$ with $[\mathrm{SiOH}]=4.84 \mathrm{~nm}^{-2}+2.0 \mathrm{~nm}$ vacuum & 2D-slab & -1.49 & 5.97 \\
\hline $\mathrm{Q}^{3} \mathrm{Q}^{4}$ surface $\mathrm{SiO}_{2}$ with $[\mathrm{SiOH}]=6.86 \mathrm{~nm}^{-2}+2.0 \mathrm{~nm}$ vacuum & 2D-slab & -1.25 & 5.99 \\
\hline Slab of liquid water 150 molecules $+2.0 \mathrm{~nm}$ vacuum & 2D-slab & $-0.12 \pm 0.30$ & $6.04 \pm 0.39$ \\
\hline Slab of liquid water 200 molecules $+2.0 \mathrm{~nm}$ vacuum & 2D-slab & $-0.03 \pm 0.37$ & $5.53 \pm 0.59$ \\
\hline Bulk water & Experiment & $-0.12^{75}$ & $6.9^{74}$ \\
\hline Bulk a-PE chains with ghost atoms separated by $0.148 \mathrm{~nm}$ & 3D-periodic with LDA in CP2k & $-0.10 \pm 0.37$ & $7.66 \pm 0.07$ \\
\hline Bulk a-PE rings with ghost atoms separated by $0.148 \mathrm{~nm}$ & $3 \mathrm{D}$-periodic with LDA in $\mathrm{CP} 2 \mathrm{k}$ & $-0.14 \pm 0.37$ & $7.58 \pm 0.07$ \\
\hline Bulk a-PE chains with ghost atoms separated by $0.148 \mathrm{~nm}$ & 3D-periodic with revised PBE in CP2k & $0.04 \pm 0.38$ & $7.72 \pm 0.05$ \\
\hline Bulk a-PE rings with ghost atoms separated by $0.148 \mathrm{~nm}$ & 3D-periodic with revised PBE in CP2k & $-0.14 \pm 0.08$ & $7.58 \pm 0.07$ \\
\hline
\end{tabular}
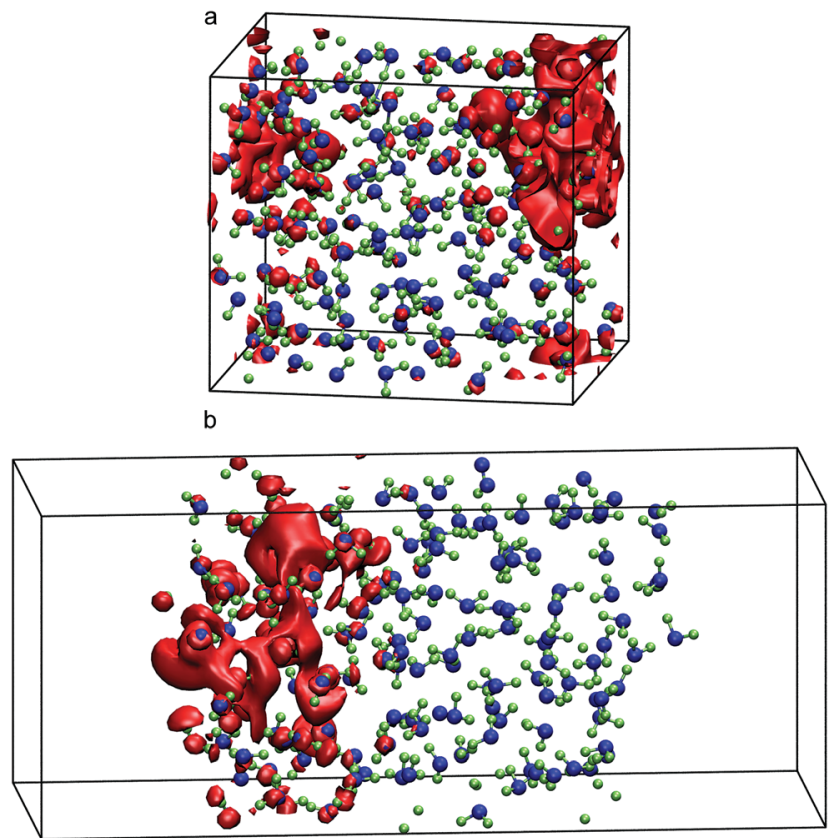

Fig. 2 Red isosurfaces show $80 \%$ of the excess electron charge with values of $1.67 \times 10^{-4}$ electron per Bohr ${ }^{3}$ in bulk (a) and $2.59 \times 10^{-4}$ electron per Bohr ${ }^{3}$ with a $2.0 \mathrm{~nm}$ vacuum gap (b) of the LUMO of the neutral system of 150 molecules of bulk liquid water. Oxygen atoms are painted in blue and hydrogens in green.

phase. Fig. 4 shows that in these systems the electron prefers to sit on a water region near the interface with polyethylene similar to the water/vacuum case. This result is consistent with the nature of polyethylene, a compound that repels injected negative charge carriers due to its negative electron affinity.

Although we cannot provide LUMO energies, we believe that the impact of the polymer on the localisation and energy of the solvated electron should be small and therefore, we can assume that its energy can be taken from the 2D-slab calculations of water and vacuum. Furthermore, this two-component system

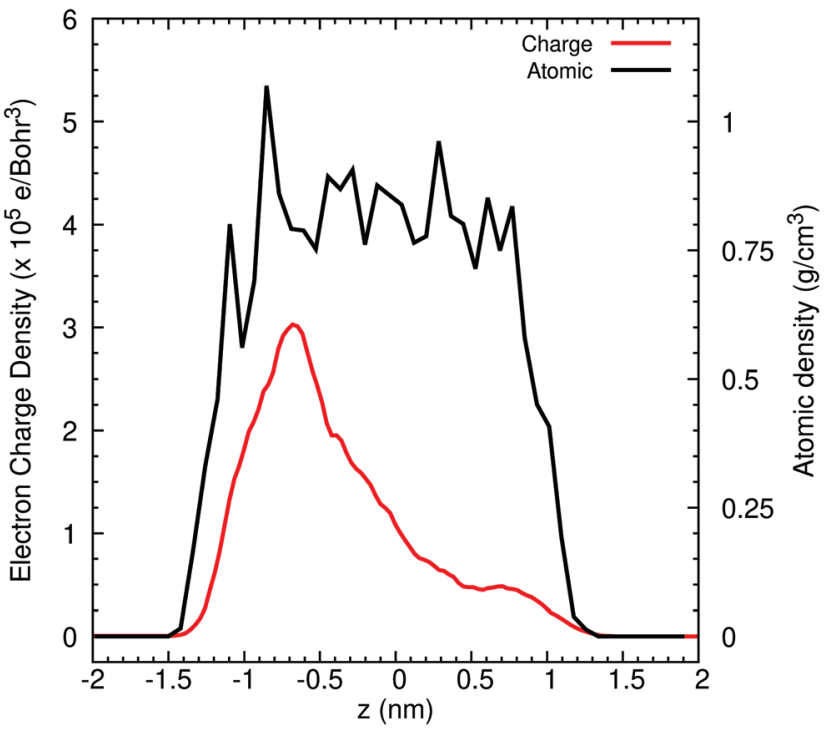

Fig. 3 Averaged profiles of the LUMO charge and atomic densities across the $z$ direction in a water/vacuum system with 150 molecules for different time intervals of $a b$ initio molecular dynamics simulation.

offers us the possibility of studying the transport of the extra charge across the interfaces via calculating the valence (VBO) and conduction band ( $\mathrm{CBO}$ ) offsets. The former are determined using the method of layer-decomposed density of states proposed by Shi and Ramprasad ${ }^{70}$ since the traditional line-up method $^{71}$ is more appropriate for crystalline systems as it assumes that the variation of the electrostatic potential is only affected by the changes of the outmost atomic layers assembled at the interface formed by two compounds. In contrast, in our work, the potential will also change due to the lack of periodic arrangement of the atomic positions in our amorphous and liquid systems. This aperiodicity obliges to us to apply the method of layer-decomposed density of states for a number of configurations (obtained every $0.25 \mathrm{ps}$ ) to obtain robust statistical results. In each configuration, we calculate the density of 


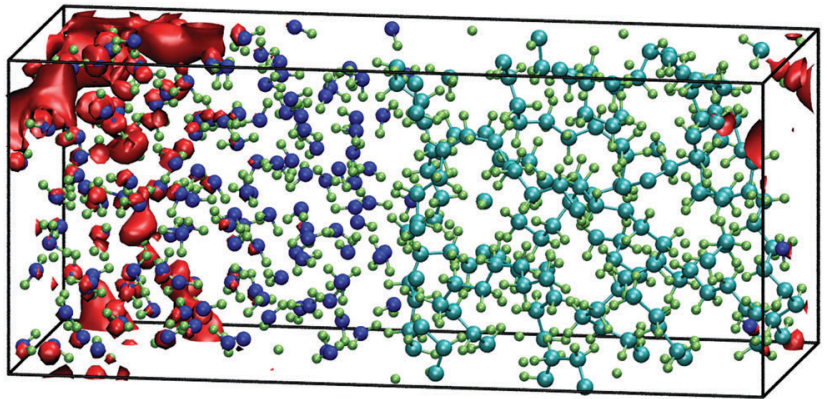

Fig. 4 Red isosurfaces show an electron charge density of $1.65 \times$ $10^{-4}$ electron per Bohr ${ }^{3}$ of the LUMO of the interfacial system of water and amorphous polyethylene at $15 \mathrm{ps}$ of a 3D-periodic ab initio simulation that encloses $80 \%$ of the excess electron charge.

states with CRYSTAL17 in 15 slices of atoms with a thickness of $0.26 \mathrm{~nm}$ and from each slice spectrum we find the HOMO. We plot the HOMOs of each layer $v s$. its position perpendicular to the interface (e.g. $z$ ) and the valence band offset is taken as the maximum difference between the HOMOs in water and polyethylene (see Fig. S4 in the ESI $\dagger$ ):

$$
\mathrm{VBO}=\max \left(E_{\mathrm{HOMO}, \mathrm{a}-\mathrm{PE}}-E_{\mathrm{HOMO}, \text { water }}\right),
$$

where $E_{\mathrm{HOMO}, \mathrm{a}-\mathrm{PE}}$ is the energy of the HOMO in a slice of the amorphous polyethylene slab and $E_{\text {HOMO,water }}$ is the energy of the HOMO in a division of the water ensemble. Fig. 5(a) shows the valence band offset of an interfacial system of water and amorphous polyethylene $v s$. time between 0 and 12 ps. After an initial drop between 0 and 1 ps, the offset oscillates between 0.1 and $1.1 \mathrm{eV}$ with an average value of $0.58( \pm 0.23) \mathrm{eV}$ between 1.0 and $12.0 \mathrm{ps}$. In addition, this property is positive at all times, which indicates that holes will move from water to polyethylene. The large fluctuations arise from the variation in the bulk water HOMO $-7.49( \pm 0.18) \mathrm{eV}$ rather than the bulk polymer $-7.25( \pm 0.06) \mathrm{eV}$ (from 3D-slab CRYSTAL17 simulations) as shown in Fig. 5(b). Clearly, the configurational fluctuations associated with the liquid water phase introduce large statistical fluctuations in the VBO. A phenomenon that will be present wherever we have a water/solid interface.

Having determined the valence band offsets, we now calculate the conduction band offsets (CBO) which are defined as

$$
\mathrm{CBO}=\mathrm{VBO}+\left(E_{\mathrm{g}, \mathrm{a}-\mathrm{PE}}-E_{\mathrm{g}, \text { water }}\right)
$$

where $E_{\mathrm{g}, \mathrm{a}-\mathrm{PE}}$ and $E_{\mathrm{g} \text {,water }}$ are the band gap energies of amorphous polyethylene $(8.01 \mathrm{eV}$ taken from averaging between 1 and 12 ps a bulk simulation without ghost atoms) and water (taken the experimental value of $6.9 \mathrm{eV}$ from ref. 74). Inserting these two energies in eqn (4), we find that the average CBO 1.69 $( \pm 0.23)$ eV between 1.0 and 12.0 ps. This result suggests that an extra electron will require $1.69 \mathrm{eV}$ to move from the liquid to the polymer, the water acting as a deep trap with respect to the polymer (for the trap depth with respect to the vacuum, see below). This conclusion is consistent with the localisation picture seen in Fig. 4 and reflects the negative electron affinity of the polymer.
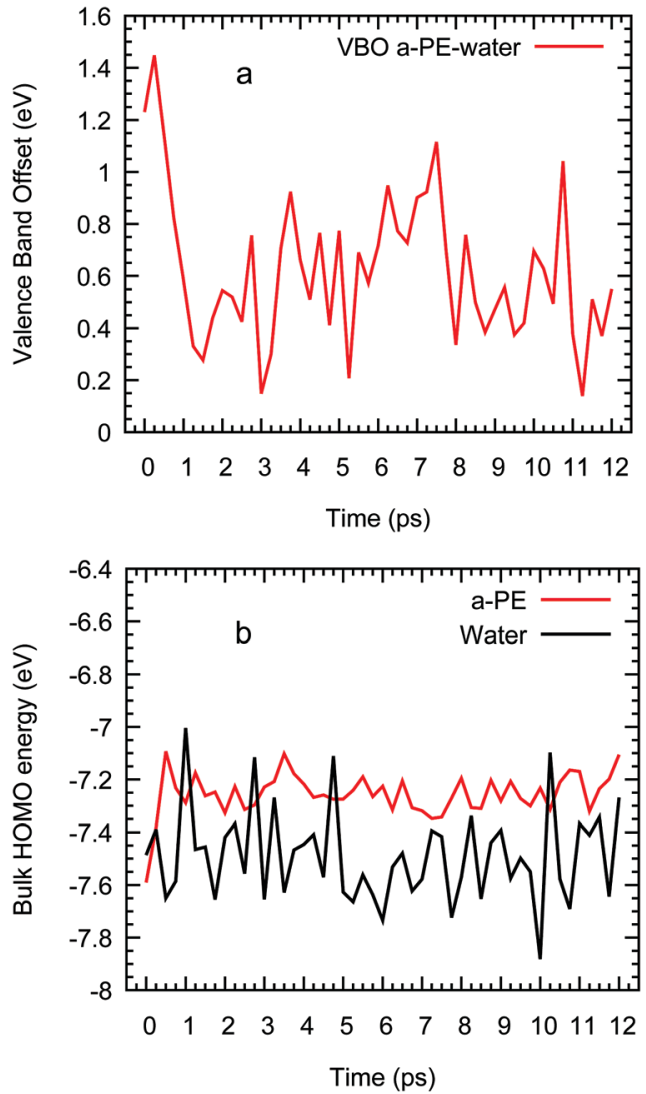

Fig. 5 Valence band offset of an interfacial system of water and amorphous polyethylene vs. time (a) and HOMO of bulk water and polyethylene (b) from ab initio molecular simulations.

\section{3. Interfaces of amorphous polyethylene and silica}

The isosurface at $80 \%$ of an excess electron charge represented by the LUMO from a 3D-periodic calculation with amorphous polyethylene and silica is illustrated for a $Q^{4}$ surface in Fig. 6(a) and for a $\mathrm{Q}^{3} / \mathrm{Q}^{4}$ surface with a silanol concentration of $6.86 \mathrm{~nm}^{-2}$ in Fig. 6(b) after 7.5 of a CP2k run with PBEsol. These two isosurfaces show that the addition of $\mathrm{OH}$ groups on the surface makes the excess electron migrate to the inner layers of the silica, which is demonstrated quantitatively in the average charge profile drawn in Fig. 6(c) for all five silanol concentration. In contrast, when we impose a vacuum on both sides of the interfacial system, the excess electron sits at the silica/polyethylene interface for any finite silanol concentration, see the isosurfaces in Fig. 7(a) and (b) and the average charge profiles in Fig. 7(c) after 7.5 of a CP2k run with LDA to avoid polymer evaporation.

In addition, we also compare the valence band-offsets obtained from the 3D-periodic and 2D-slab $\mathrm{Q}^{4}$ calculations. We obtain $1.14( \pm 0.19) \mathrm{eV}$ for the $3 \mathrm{D}$-periodic case and 0.84 $( \pm 0.36) \mathrm{eV}$ for the $2 \mathrm{D}$-slab case. Using eqn (4) to calculate the $\mathrm{CBO}$ in this system by (experimental silica's band gap of $8.8 \mathrm{eV}$ and the DFT amorphous polyethylene's of $8.01 \mathrm{eV}$ ) the CBOs will be $0.79 \mathrm{eV}$ lower than the VBOs, at $0.33 \mathrm{eV}$ for the $3 \mathrm{D}$-periodic and $0.05 \mathrm{eV}$ for the 2D-slab. The latter figure consistent with the 


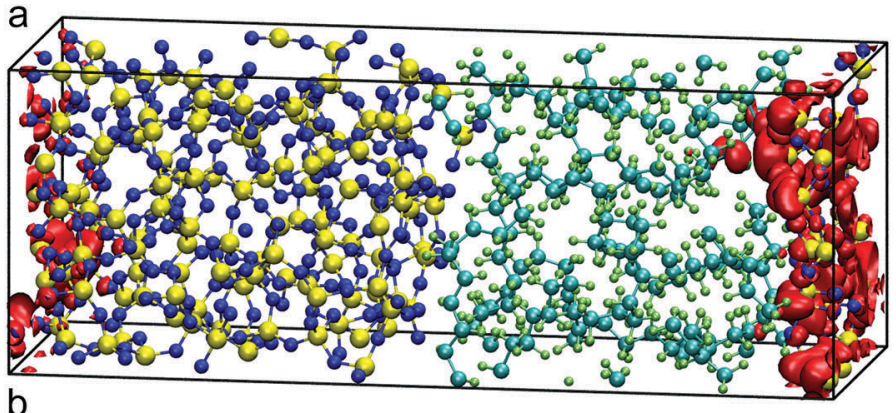

b

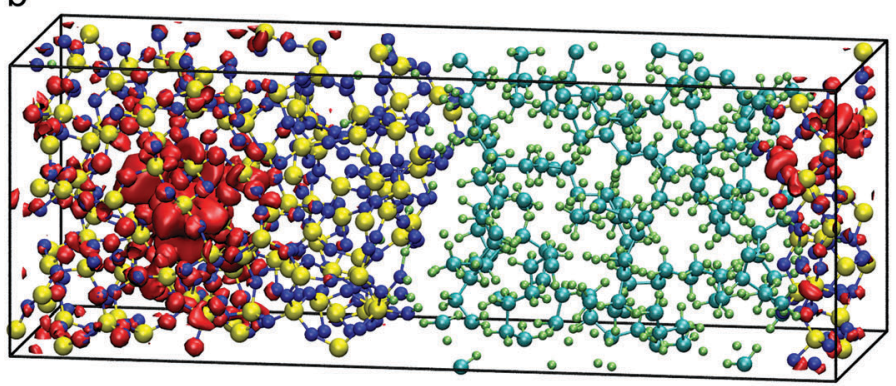

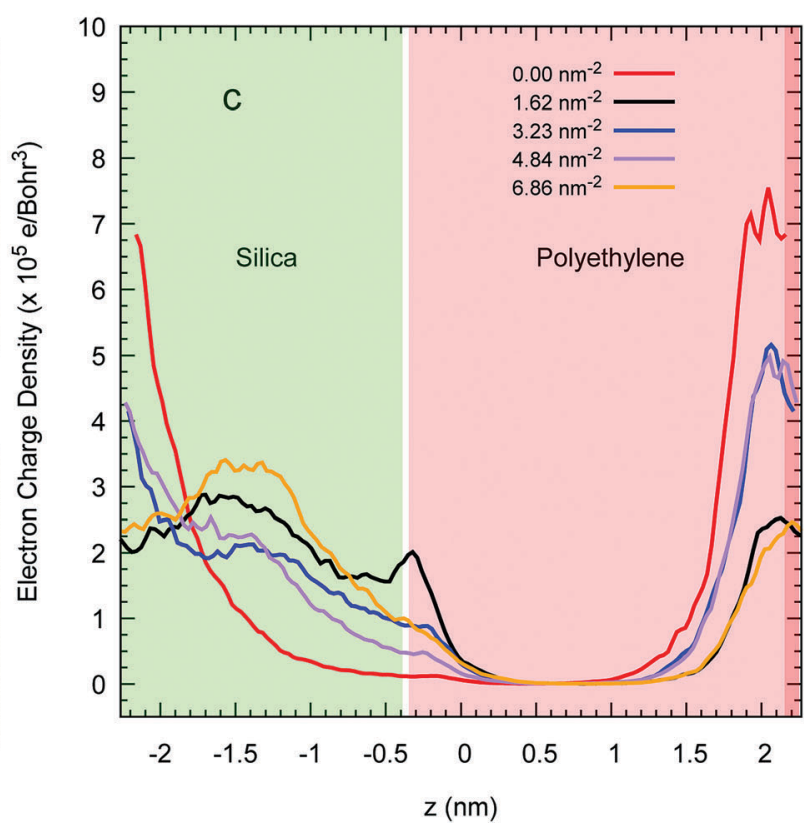

Fig. 6 Panels (a) and (b) illustrate red isosurfaces with an electron charge density of $1.442 \times 10^{-4}$ and $1.077 \times 10^{-4}$ electron per Bohr ${ }^{3}$, respectively, of the $80 \%$ of the LUMO charge of the interfacial system of polyethylene (right) and amorphous silica (left) whose surfaces are $Q^{4}$ (a) and $Q^{3} / Q^{4}$ with a silanol concentration of $6.86 \mathrm{~nm}^{2}$ (b) at 7.5 ps drawn from 3D simulations with no vacuum. Panel (c) plots the average longitudinal profile of the LUMO's charge density for a number of silanol concentrations.

a



b

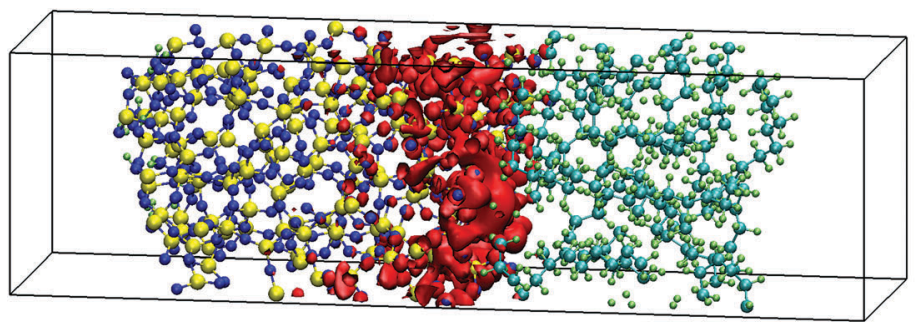

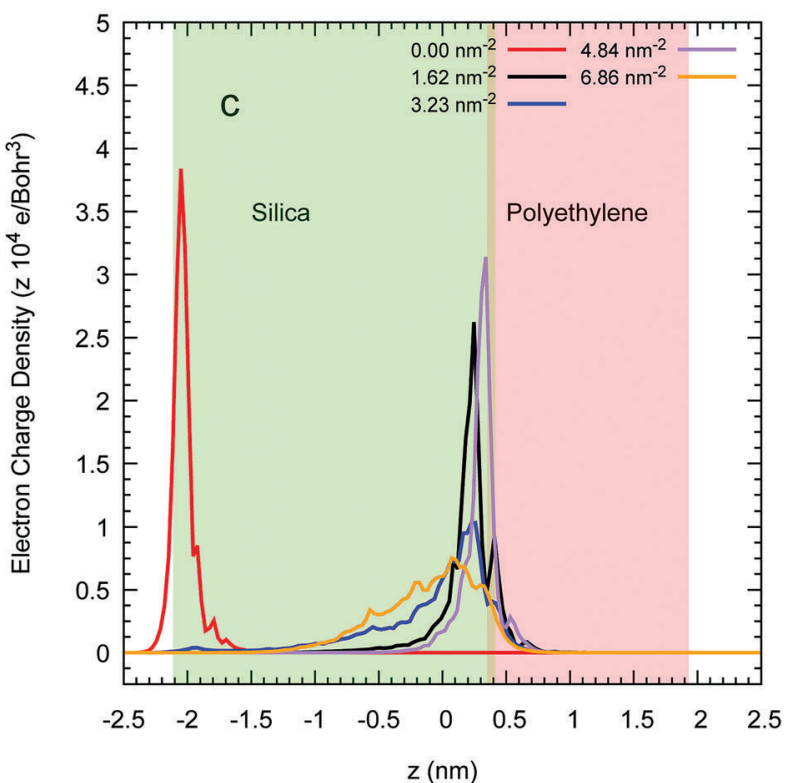

$\mathrm{z}(\mathrm{nm})$

Fig. 7 Panels (a) and (b) illustrate red isosurfaces with an electron charge density of $51.52 \times 10^{-4}$ and $1.96 \times 10^{-4}$ electron per Bohr ${ }^{3}$ of the $80 \%$ of the LUMO' charge of the interfacial system of polyethylene (right) and amorphous silica (left) whose surfaces are $Q^{4}$ (a) and $Q^{3} / Q^{4}$ with a silanol concentration of $6.86 \mathrm{~nm}^{2}$ (b) at 7.5 ps drawn from 2D SLAB simulations with vacuum. Panel (c) plots the average longitudinal profile of the LUMO's charge density for a number of silanol concentrations. Space not painted in colour represents vacuum.

dominant role of the interfaces in determining excess electron behaviour in the $2 \mathrm{D}$ slab.

For these 2D-slab cases, we plot in Fig. 8 the LUMO energies vs. silanol concentrations after correcting with respect to the vacuum level. Our results show that the energy of the excess electron increases with increasing concentration. We argue that this increase is caused by the increasing delocalisation of this orbital. The most negative energy is $-1.75 \mathrm{eV}$ with the $\mathrm{Q}^{4}$ surface, which is consistent with the value of $-1.54 \mathrm{eV}$ obtained when this slab is isolated with vacuum on both sides. When the silanol content increases, the LUMO position transits from the left interface to the right wall with some charge penetrating 


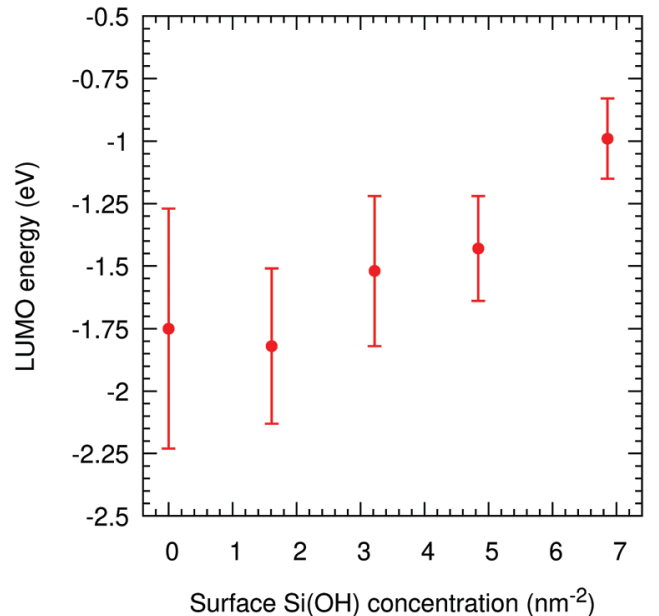

Fig. 8 LUMO energies in amorphous polyethylene and silica vs. the silanol concentration on the surface for 2D-SLAB calculations.

into the polyethylene. The hydroxylated silica/PE interfaces act as deep traps with energies between $-1.75 \mathrm{eV}$ and $-0.99 \mathrm{eV}$ (but see the next section for what happens if free water is present).

\section{4. Interfaces of amorphous silica and water}

We now study excess electrons in systems made with an amorphous silica slab sandwiched with two ensembles of 150 water molecules on both sides as shown in Fig. 9 using the revPBE functional. These systems do not need to include polyethylene slabs which will repel the extra charge due to the negative electron affinity of this polymer. This behaviour is shown in water/polyethylene and silica/polyethylene interfaces, where the excess electron sits away from the polymer, which has little effect on its degree of localisation and energy. This water/silica/water system is run with CP2k and revPBE for $15 \mathrm{ps,}$ during which the interfacial chemistry spontaneously produces four silanols on each silica wall equivalent to a silanol surface concentration of $1.62 \mathrm{~nm}^{-2}$. This concentration is slightly higher than the $1.37 \mathrm{~nm}^{-2}$ reported on nanoporous silica surfaces modelled with an NVT molecular dynamics for 30 ps using CP2k and the BLYP functional. ${ }^{72}$ However, both values underestimate the experimental range between 4.2 and $5.7 \mathrm{~nm}^{-2}$ collected in ref. 53 for a range of different treatments to rehydroxylate silica surfaces. This disagreement is expected given the different length and time scales in simulations and experiments.
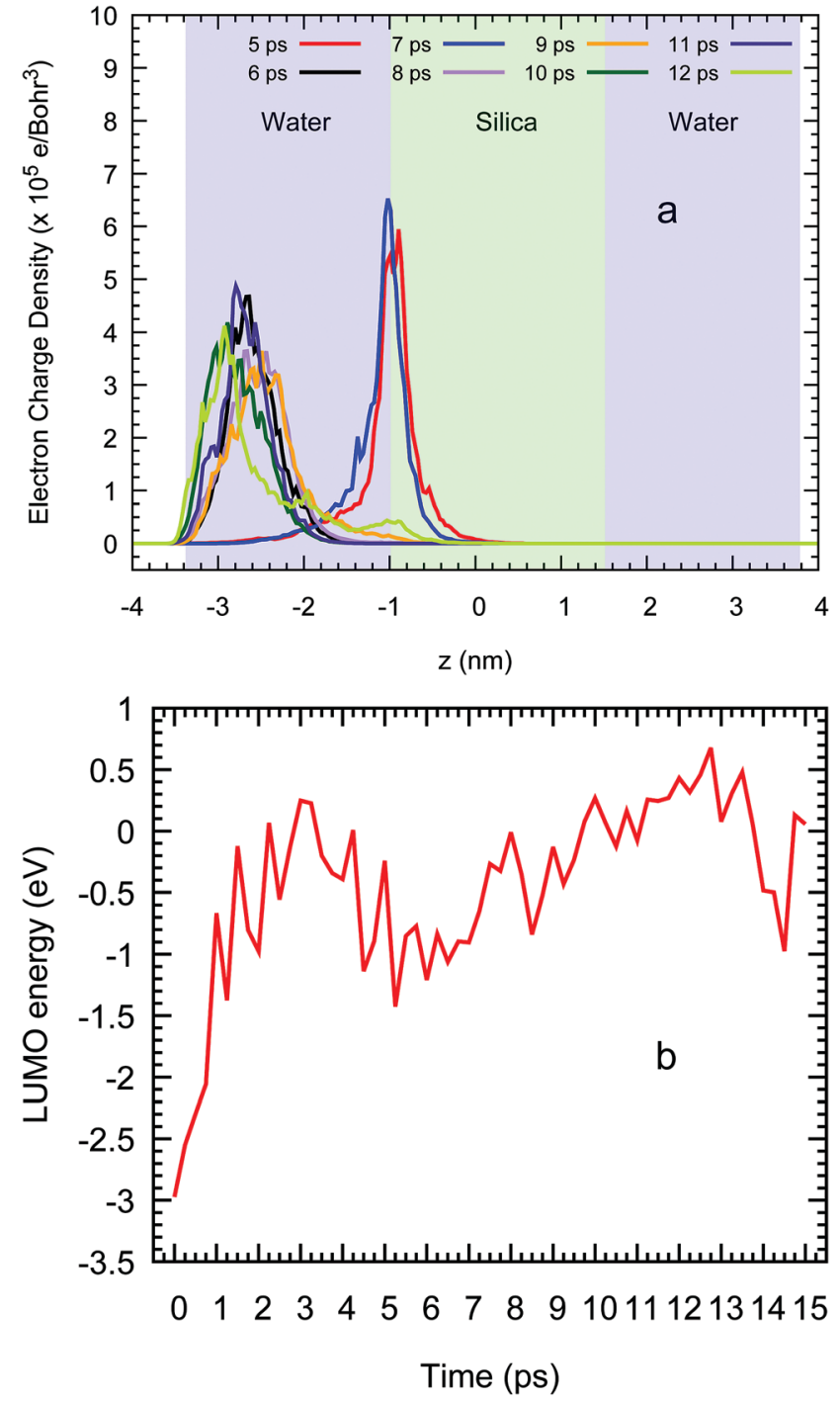

Fig. 10 (a) Average profile of the LUMO charge density across the $z$ direction and (b) the energy of this orbital vs. the ab initio simulation time in a water/silica/water system.

Once the system is well stabilised after $7 \mathrm{ps,} \mathrm{the} \mathrm{spatial}$ localisation of the excess electron shows a very similar picture as in the water/vacuum case given that this particle again sits near the vacuum on the left liquid ensemble as shown at several CP2k simulation times in Fig. 10(a). Moreover, this same behaviour is also seen in the LUMO energies of this

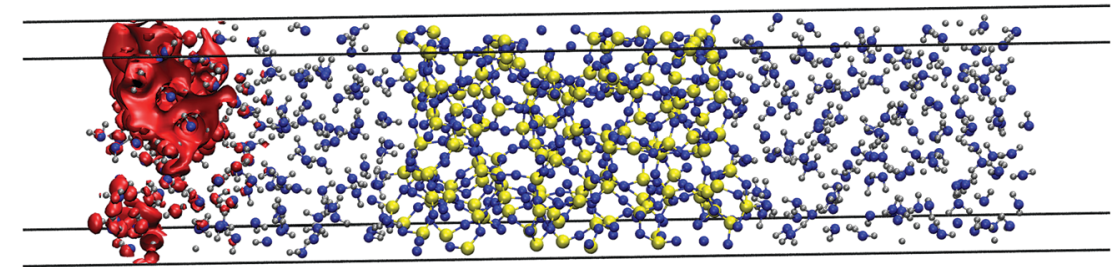

Fig. 9 Red isosurface of $2.42 \times 10^{-4}$ electron per Bohr ${ }^{3}$ shows the position of the $80 \%$ of the LUMO's charge at 10 ps of a system made with amorphous silica and two systems of 150 water molecules. 
system in Fig. 10(b), which show an increase as water density slightly decreases and silanol groups are formed on both surfaces with an average value of $-0.27( \pm 0.53) \mathrm{eV}$ between 4 and 15 ps. With a standard deviation nearly two times higher than the absolute value of the average, our DFT methodology predicts that the excess electron in this system oscillates around the same average energy as in the case of a vacuum/ water systems, leading us to conclude that, in the presence of water, the detailed interfacial chemistry of the silica surface does not influence the behaviour of the excess electron in nanocomposite materials.

\section{Conclusion}

We have calculated the energy and degree of localisation of excess electrons at a number of interfaces made by combining amorphous polyethylene, silica, and liquid water to be representative of interfaces to be found in wet and dry polymer nanocomposites. For pure silica we find an excess electron would be strongly localised with an energy of around $-1.5 \mathrm{eV}$. Hydroxylating the silica surface and adding a polyethylene interface produces a strong dependence of the excess electron behaviour on the silanol concentration. An excess electron sits at the interface with an energy of between $-1.75 \mathrm{eV}$ for the $\mathrm{Q}^{4}$ surface chemistry and $-0.99 \mathrm{eV}$ for the $\mathrm{Q}^{3} / \mathrm{Q}^{4}$, becoming less localised with increasing silanol density. However, in the presence of a water film, the detailed interfacial chemistry of the silica surface becomes irrelevant and the excess electron sits preferentially at the water/vapour interface with an energy of minus a few tenths of an $\mathrm{eV}$. We conclude that the moisture content of a polymer nanocomposite has a profound influence on the electron trapping behaviour with a wet interfacial material producing much lower trapping energies and a high excess electron mobility (from the Einstein relation). We would then expect that a wet nanocomposite with a percolating water film would show increased electrical conductivity, independent of surface chemistry, which is consistent with our experimental data for low density polyethylene octylnanosilica composites. ${ }^{32}$

\section{Conflicts of interest}

There are no conflicts to declare.

\section{Acknowledgements}

The authors acknowledge the financial support of EPSRC through grant EP/N002288/1, Advanced Polymer Material for Energy Security - POLYMAT and HPC support through ARCHER project e446. This work was supported by EPSRC through a Service Level Agreement with STFC Scientific Computing Department and by the UK Materials Chemistry Consortium (Grant EP/L000202). NQ thanks Alun Vaughan for useful discussions.

\section{References}

1 C. J. Lombardo, M. S. Glaz, Z.-E. Ooi, D. A. Vanden Bout and A. Dodabalapur, Phys. Chem. Chem. Phys., 2012, 14, 13199-13203.

2 C. Groves, J. C. Blakesley and N. C. Greenham, Nano Lett., 2010, 10, 1063-1069.

3 L. G. Kaake, P. F. Barbara and X. Y. Zhu, J. Phys. Chem. Lett., 2010, 1, 628-635.

4 M. A. Henderson, J. M. White, H. Uetsuka and H. Onishi, J. Am. Chem. Soc., 2003, 125, 14974-14975.

5 Y. Okahata, T. Kobayashi, K. Tanaka and M. Shimomura, J. Am. Chem. Soc., 1998, 120, 6165-6166.

6 R. Rinaldi, E. Branca, R. Cingolani, R. Di Felice, A. Calzolari, E. Molinari, S. Masiero, G. Spada, G. Gottarelli and A. Garbesi, Ann. N. Y. Acad. Sci., 2002, 960, 184-192.

7 P. F. Barbara, T. J. Meyer and M. A. Ratner, J. Phys. Chem., 1996, 100, 13148-13168.

8 B. L. Mahaney and K. Meek, J. Biochem., 2009, 417, 639-650.

9 Z. G. Yu and X. Song, Phys. Rev. Lett., 2001, 86, 6018-6021.

10 Around $9 \%$ of the generated electricity is lost through transmission and distribution, amounting to an estimated financial loss of 3 trillion U.S. dollars a year worldwide according to the International Electrotechnical Commission: http://www.iec.ch/, accessed February 2018.

11 B. Chu, X. Zhou, K. Ren, B. Neese, M. Lin, Q. Wang, F. Bauer and Q. M. Zhang, Science, 2006, 313, 334-336.

12 V. Sharma, C. Wang, R. G. Lorenzini, R. Ma, Q. Zhu, D. W. Sinkovits, G. Pilania, A. R. Oganov, S. Kumar, G. A. Sotzing, S. A. Boggs and R. Ramprasad, Nat. Commun., 2014, 5, 4845 .

13 T. D. Huan, S. Boggs, G. Teyssedre, C. Laurent, M. Cakmak, S. Kumar and R. Ramprasad, Prog. Mater. Sci., 2016, 83, 236-239.

14 M. H. Yoon, H. Yan, A. Facchetti and T. J. Marks, J. Am. Chem. Soc., 2005, 127, 10388-10395.

15 M. E. Roberts, N. Queraltó, S. C. B. Mannsfeld, B. N. Reinecke, W. Knoll and Z. Bao, Chem. Mater., 2009, 21, 2292-2299.

16 M. A. Hickner, H. Ghassemi, Y. S. Kim, B. R. Einsla and J. E. McGrath, Chem. Rev., 2004, 104, 4587-4612.

17 Y. J. Wang, J. Qiao, R. Baker and J. Zhang, Chem. Soc. Rev., 2013, 42, 5768-5787.

18 S. Dadbin, M. Frounchi, M. H. Saeid and F. Gangi, J. Appl. Polym. Sci., 2002, 86, 1959-1969.

19 L. A. Dissado and J. C. Fothergill, Electrical Degradation and Breakdown in Polymers, P. Peregrinus, London, 1992.

20 W. L. Hawkins, Polymer Degradation and Stabilization, Springer-Verlag, Berlin, 1984.

21 See review on silicate nanocomposites by S. S. Ray and M. Okamoto, Prog. Polym. Sci., 2003, 28, 1539-1641.

22 See review by Y. Tanaka, N. Ohnuma, K. Katsunami and Y. Ohki, IEEE Trans. Electr. Insul., 1991, 26, 258-265.

23 Y. Rao and C. P. Wong, J. Appl. Polym. Sci., 2004, 92, 2228-2231.

24 R. C. Smith, C. Liang, M. Landry, J. K. Nelson and L. S. Schadler, IEEE Trans. Dielectr. Electr. Insul., 2008, 15, 187-196. 
25 Y. Wang, D. MacKernan, D. Cubero, D. Coker and N. Quirke, J. Chem. Phys., 2014, 140, 154902.

26 M. Meunier, A. Aslanides and N. Quirke, J. Chem. Phys., 2001, 115, 2876-2881.

27 X. Huang, P. Jiang and Y. Yin, Appl. Phys. Lett., 2009, 95, 242905.

28 C. M. Osburn and D. W. Ormond, J. Electrochem. Soc., 1972, 119, 591-597.

29 V. V. Afanas'ev and A. Stesmans, Phys. Rev. Lett., 1997, 78, 2437.

30 Defects in Microelectronic Materials and Devices, ed. D. M. Fleetwood, S. T. Pantelides and R. D. Schrimpf, CRC Press, Boca Raton, 2009.

31 T. Y. Chan, K. K. Young and C. Hu, IEEE Electron Device Lett., 1987, 8, 93-95.

32 S. Virtanen, A. S. Vaughan, L. Yang, F. Saiz and N. Quirke, Dielectric Breakdown Strength and Electrical Conductivity of Low Density Polyethylene Octylnanosilica Composite, IEEE Conference on Electrical Insulation and Dielectric Phenomena (IEEE CEIDP), IEEE, pp. 58-61, ISSN: 00849162, 2016, see also S. Virtanen, A. S. Vaughan, L. Yang, F. Saiz and N. Quirke, Electrical Conductivity and Moisture Uptake Studies of Low Density Polyethylene Octyl-nanosilica Composite, conference paper, 25th Nordic Insulation Symposium on Materials, Components and Diagnostics, 2017.

33 M. Meunier and N. Quirke, J. Chem. Phys., 2000, 113, 369-376.

34 D. Cubero, N. Quirke and D. F. Coker, J. Chem. Phys., 2003, 119, 2669-2679.

35 D. Cubero and N. Quirke, J. Chem. Phys., 2004, 120, 7772-7778.

36 M. C. Righi, S. Scandolo, S. Serra, S. Iarlori, E. Tosatti and G. Santoro, Phys. Rev. Lett., 2001, 87, 076802.

37 M. Unge, T. Christen and C. Törnkvist, Electronic structure of polyethylene-Crystalline and amorphous phases of pure polyethylene and their interfaces, Annual Report Conference on Electrical Insulation and Dielectric Phenomena (CEIDP), IEEE, 2012, pp. 525-530.

38 S. Serra, S. Iarlori, E. Tosatti, S. Scandolo, M. C. Righi and G. E. Santoro, Chem. Phys. Lett., 2002, 360, 487-493.

39 M. Unge and T. Christen, Chem. Phys. Lett., 2014, 613, 15-18.

40 R. Stowasser and R. Hoffmann, J. Am. Chem. Soc., 1999, 121, 3414-3420.

41 F. Saiz, D. Cubero and N. Quirke, Phys. Chem. Chem. Phys., 2018, DOI: 10.1039/C8CP01330F.

42 L. T. Zhuravlev, Colloids Surf., A, 2000, 173, 1-38.

43 M. Schneemilch and N. Quirke, J. Chem. Phys., 2018, 148, 194704.

44 S. J. Plimpton, J. Comput. Phys., 1995, 117, 1-19.

45 R. J. Hemley, A. P. Jephcoat, H. K. Mao, L. C. Mingt and M. H. Manghnanit, Nature, 1988, 334, 52-54.

46 Dassault Systèmes BIOVIA, Materials Studio Suite, v.5.5.2, San Diego, Dassault Systèmes, 2017.

47 H. Sun, J. Phys. Chem. B, 1998, 102, 7338-7364.

48 J. Hutter, M. Iannuzzi, F. Schiffmann and J. VandeVondele, Comput. Mol. Sci., 2014, 4, 15-25.
49 J. VandeVondele, M. Krack, F. Mohamed, M. Parrinello, T. Chassaing and J. Hutter, Comput. Phys. Commun., 2005, 167, 103-128.

50 S. Goedecker, M. Teter and J. Hutter, Phys. Rev. B: Condens. Matter Mater. Phys., 1996, 54, 1703.

51 M. Krack, Theor. Chem. Acc., 2005, 114, 145-152.

52 S. Grimme, J. Comput. Chem., 2006, 27, 1787-1799.

53 T. P. M. Goumans, A. Wander, W. A. Brown and C. R. A. Catlow, Phys. Chem. Chem. Phys., 2007, 9, 2146-2152.

54 L. T. Zhuralev, Langmuir, 1987, 3, 316-318.

55 M. Dion, H. Rydberg, E. Schröder, D. C. Langreth and B. I. Lundqvist, Phys. Rev. Lett., 2005, 92, 246401.

56 J. L. Abascal and C. Vega, J. Chem. Phys., 2005, 123, 234505.

57 J. P. Perdew, K. Burke and M. Ernzerhof, Phys. Rev. Lett., 1986, 77, 3865-3868.

58 B. Hammer, L. B. Hansen and J. K. Nørskov, Phys. Rev. B: Condens. Matter Mater. Phys., 1999, 59, 7413.

59 J. P. Perdew, A. Ruzsinszky, G. I. Csonka, O. A. Vydrov, G. E. Scuseria, L. A. Constantin, X. Zhou and K. Burke, Phys. Rev. Lett., 2008, 100, 136406.

60 R. Dovesi, R. Orlando, A. Erba, C. M. Zicovich-Wilson, B. Civalleri, S. Casassa, L. Maschio, M. Ferrabone, M. De La Pierre, P. D’Arco, Y. Noel, M. Causa, M. Rerat and B. Kirtman, Int. J. Quantum Chem., 2014, 114, 1287-1317.

61 G. A. Petersson, A. Bennett, T. G. Tensfeldt, M. A. Al-Laham, W. A. Shirley and J. Mantzaris, J. Chem. Phys., 1988, 89, 2193-2218.

62 G. A. Petersson and M. A. Al-Laham, J. Chem. Phys., 1991, 94, 6081-6090.

63 E. G. Lewards, Computational Chemistry, Springer, New York, 2nd edn, 2011, ch. 5, pp. 232-255.

64 Al-M. El-Sayed, M. B. Watkins, V. V. Afanas'ev and A. L. Shluger, Phys. Rev. B: Condens. Matter Mater. Phys, 2014, 89, 125201.

65 M. Chiesa, M. C. Paganini and E. Giamello, Chem. Phys. Chem., 2004, 5, 1897-1900.

66 R. E. Larsen, W. J. Glover and B. J. Schwartz, Science, 2010, 329, 65-69.

67 K. H. Schmidt, P. Han and D. M. Bartels, J. Phys. Chem., 1992, 96, 199-206.

68 L. Turi, Á. Madarász and P. J. Rossky, J. Chem. Phys., 2006, 125, 014308.

69 M. Chen, H.-Y. Ko, R. C. Remsing, M. F. Calegari Andrade, B. Santra, Z. Sun, A. Selloni, R. Car, M. L. Klein, J. P. Perdew and X. Wu, Proc. Natl. Acad. Sci. U. S. A., 2017, 114, 10846-10851.

70 N. Shi and R. Ramprasad, IEEE Trans. Dielectr. Electr. Insul., 2008, 15, 170-177.

71 C. G. Van de Walle and R. M. Martin, Phys. Rev. B: Condens. Matter Mater. Phys., 1987, 35, 8154-8165.

72 J. M. Rimsza, J. Yeon, A. C. T. van Duin and J. Du, J. Phys. Chem. C, 2016, 120, 24803-24816.

73 Taken as the electron affinity from the photoemission experiments made by V. W. Ballarotto, M. Breban, K. Siegrist, R. J. Phaneuf and E. D. Williams, J. Vac. Sci. 
Technol., B, 2002, 20, 2514-2518 on devices covered by ultrathin $\mathrm{SiO}_{2}$ layers.

74 S. W. King, J. Brockman, M. French, M. Jaehnig, M. Kuhn and B. French, J. Appl. Phys., 2014, 116, 113703.

75 J. V. Coe, A. D. Earhart, M. C. Cohen, G. J. Hoffman, H. W. Sarkas and K. H. Bowen, J. Chem. Phys., 1997, 107, 6023-6031. See also J. V. Coe, Int. Rev. Phys. Chem., 2001, 20, 33-58. This value of $6.9 \mathrm{eV}$ is in good agreement by $7.3 \mathrm{eV}$ obtained with start-of-the-art GW calculations by C. Fang, W.-F. Li, R. S. Koster, J. Klimeš, A. van Blaaderen and M. A. van Huis, Phys. Chem. Chem. Phys., 2015, 17, 365-375. 76 K. J. Less and E. G. Wilson, J. Phys. C: Solid State Phys., 1973, 6, 3110-3120.

77 Measured value for the alkane $\mathrm{C}_{36} \mathrm{H}_{74}$ of $8.8 \mathrm{eV}$ by E. Cartier and P. Pfluger, Phys. Rev. B: Condens. Matter Mater. Phys, 1986, 34, 8822-8827. 